\title{
A socio-emotional model of impoliteness for Non-Player Characters
}

\section{(Extended Abstract)}

\author{
Sabrina Campano \\ Université Pierre et Marie Curie \\ 4 , place Jussieu \\ 75252, Paris, France \\ sabrina.campano@etu.upmc.fr
}

\author{
Nicolas Sabouret \\ Laboratoire Informatique de Paris 6 \\ LIP6, 104 av du Président Kennedy \\ 75016, Paris, France \\ nicolas.sabouret@lip6.fr
}

\section{INTRODUCTION}

Due to its important role in dialogue, politeness has been widely studied both from a theoretical point of view and at a practical level in virtual agents [1, 4]. However, few attention has been given to impoliteness, even though virtual characters, such as Non Player Characters in video games, should be able to use it to improve their believability. Impoliteness consists in performing verbal attacks towards the interlocutor [3], most of the time deliberately, while politeness consists in minimizing verbal offenses towards the interlocutor [2].

Our work aims to provide an impoliteness model for Non Player Characters in video games, with the objective to improve their believability. Non Player Characters are virtual agents controlled by the game, who interacts with other NPCs, or with Player Characters who are controlled by humans.

Like politeness, impoliteness is subjectively interpreted, and it is hard to state upon the impoliteness level of a sentence and the response it will cause without considering some individual paramaters. The individual parameters we propose to take into account are the personality and the emotions of a NPC, along with his social relations with the other characters in the game. Given an utterance set proposed to a NPC for his turn taking, we want him to select the appropriate utterance relative to impoliteness, according to his individual parameters. Our approach relies on a cognitive model, but is also inspired from biological data concerning personality and emotions. We try to infer hypotheses from those different sources in order to assemble them in a model, and to test it through experimentation.

\section{PROPOSED MODEL}

In this model impoliteness is provoked by fear and anger emotions, or depends on social relations. Personality, social relations and language register modify the influence of emotions under definite conditions described below. A language register is a variety of language used in a specific social setting. In the model it corresponds to an element of the set $\mathcal{R}=\{$ coarse, familiar, casual, consultative, formal,

Cite as: A socio-emotional model of impoliteness for Non-Player Characters, (Extended Abstract), Sabrina Campano, Nicolas Sabouret, Proc. of 8th Int. Conf. on Autonomous Agents and Multiagent Systems (AAMAS 2009), Decker, Sichman, Sierra and Castelfranchi (eds.), May, 10-15, 2009, Budapest, Hungary, pp. 1123-1124 Copyright (c) 2009, International Foundation for Autonomous Agents and Multiagent Systems (www.ifaamas.org), All rights reserved. frozen $\}$. A total order relation $\prec$ is defined on $\mathcal{R}$, such as coarse $\prec$ familiar $\prec$ casual $\prec$ consultative $\prec$ formal $\prec$ frozen.

Emotions and personality taken apart, each character $i$ NPC or PC has an expected language register for the dialogue situation, $E R_{i}(d s) \in \mathcal{R}$, which is the register to be used by $i$ in order to keep on being polite. This register is supposed to be known by $i$ and by his interlocutor. $E R_{i}(d s)$ is calculated from three values : the natural character register $N R_{i} \in \mathcal{R}$, which is the register that $i$ uses the more often, the mastered character registers $M R_{i} \subseteq \mathcal{R}$, which represents the registers that $i$ is able to use, and the imposed register for the dialogue situation $I R(d s) \in \mathcal{R} \cup\{\square\}$, which is the register that participants should use if they want to be polite. The $\square$ value represents the case there is no imposed register.

Each utterance $u$ has a language register $R(u) \in \mathcal{R}$, and an emotional vector which represents the emotions that $u$ may provoke in the interlocutor. This vector is denoted as : provoked_emotions $(u)=\left(\begin{array}{c}f \operatorname{fear}(u) \\ \operatorname{anger}(u)\end{array}\right)$

as each vector value is included in $[0,1]$. The more the value is close to 1 , the more the emotion is likely to be provoked by $u$, and the more the value is close to 0 , the less the emotion is likely to be provoked by $u$. The emotional vector for the emotions felt by a NPC $i$ at time $t$ is denoted as :

felt_emotions $_{i}(t)=\left(\begin{array}{c}\operatorname{fear}_{i}(t) \\ \operatorname{anger}_{i}(t)\end{array}\right)$

as each vector value is included in $[0,1]$. The more the value is close to 1 , the more the emotion is felt by $i$, and the more the value is close to 0 , the less the emotion is felt by $i$.

The variables anger $i(t)$ and fear $_{i}(t)$ are updated with their value at time $t-1$, respectively $\operatorname{anger}_{i}(t-1)$ and $\operatorname{fear}_{i}(t-1)$, and the emotional vector values of the last utterance $u$ that $i$ used to talk to $j$, respectively anger $(u)$ and $f e a r(u)$. Two additional variables are used to compute $\operatorname{anger}_{i}(t)$. The first is $\operatorname{liking}_{i, j}(t) \in[-1,1]$, which represents the liking value of $i$ towards $j$. The more the value is close to 1 , the more $i$ likes $j$, and the more $\operatorname{anger}_{i}(t)$ is lowered. The more the value is close to -1 , the less $i$ likes $j$, and the more $\operatorname{anger}_{i}(t)$ is increased. The second variable is the result of $r_{-} \operatorname{dif} f\left(R(u), E R_{j}(d s)\right)$, which returns the rank difference between $R(u)$, the register of the utterance pronounced by the interlocutor $j$ aimed at $i$, and $E R_{j}(d s)$, the expected register of $j$. The more the rank difference is high, the more $\operatorname{anger}_{i}(t)$ is increased. The variables fear $_{i}(t)$ 
and anger $_{i}(t)$ decrease over time if respectively fear $(u)$ and anger $(u)$ equals to 0 .

The impoliteness level computed for the NPC $i$ who talks to a character $j$ a time $t$ is represented by an impoliteness couplet $I C_{i, j}(t)=\{R \in \mathcal{R}, a \in[0,1]\}$. The first element is the register that will be used by $i$ to talk to $j$, and the second element is the anger intensity that $i$ aims to provoke in $j$. This impoliteness couplet allows to select an utterance among $\mathcal{U}_{i, j}(t)$, the utterance set proposed by an inference engine for the NPC $i$ to adress the character $j$ at time $t$. Using an utterance that can provoke anger in the interlocutor, or using a register different from the expected register is considered as impolite. When anger $_{i}(t)$ or fear $_{i}(t)$ exceeds a defined threshold, an impoliteness couplet relative to the emotion intensity value is created. The emotion intensity value taken into account is the maximum between these two variables. The more the emotion intensity is high, the more the aimed anger value $a$ is high, and the more the register $R$ is far from the expected register $E R_{j}(d s)$.

This impoliteness couplet can be modified by two other variables. The first is the agreeable personality trait of a NPC $i$, agreeableness $i \in[-1,1]$. The more the value is close to 1 , the more $i$ is agreeable, and the more $i$ aims to avoid interlocutor anger. Consequently, he tries to stay close to $E R_{i}(d s)$, and to not use an utterance provoking too much anger in the interlocutor. The other variable is dominance $_{i, j}(t) \in[-1,1]$, the dominance value of a NPC $i$ towards a character $j$. The more the value is close to 1 , the more $i$ believes to dominate $j$. The more the value is close to -1 , the more $i$ believes he is dominated by $j$, and aims to avoid interlocutor anger. Consequently, $i$ tries to stay close to $E R_{i}(d s)$, and to not use an utterance provoking too much anger in the interlocutor $j$.

In case the maximum emotion intensity value is $\operatorname{fear}_{i}(t)$, an other impoliteness couplet $I C_{i, j}(t)=\left\{E R_{i}, 0\right\}$ is created, which corresponds to the neutral behaviour of not being impolite. Then the choice between the two impoliteness couplets could be determined by the dominance $i, j(t) \in[-1,1]$ variable : if $i$ is very dominated by $j, i$ will use the neutral impoliteness couplet.

If the felt emotions intensities of a NPC $i$ are not sufficient to provoke impoliteness, a neutral impoliteness couplet $I C_{i, j}(t)=\left\{E R_{i}, 0\right\}$ is created, and if $i$ is very dominant over $j$, he can be impolite to a limited extent. Two other impoliteness couplets are created with a low aimed anger and a register which is one rank above and below the expected register.

Once that impoliteness couplets are created, the selected utterance $u$ is the one where $R(u)$ equals to the register $R$ of an impoliteness couplet, and where the difference between anger $(u)$ and the aimed anger value $a$ of this impoliteness couplet is the minimum difference, for each utterance and for each couplet.

\section{DISCUSSION AND FUTURE WORK}

We hope that our work will contribute to set up a model for impoliteness that depends on emotions, personality, and social relations. Preliminary tests made on the model seemed to reveal coherent behaviours, however an advanced evaluation is necessary to state on NPCs believability. Relatively to planification, Brown and Levinson politeness strategies [2], at the core of most of the work on politeness modelization, have to be taken into account, as the work of Culpeper

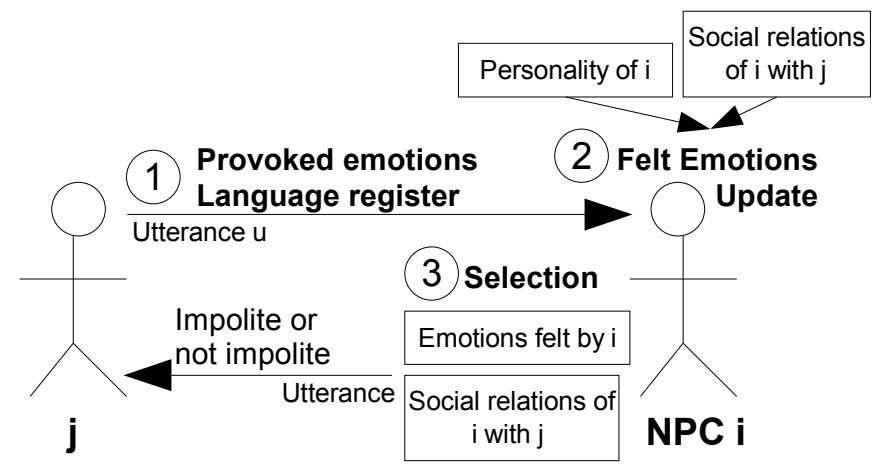

Figure 1: General principle of the model

concerning impoliteness strategies[3]. We plan to extend our model to politeness and to integrate strategies in it.

The language registers are represented in our model as discrete elements, but boundaries between registers are often imprecise. Multiple elements are involved such as vocabulary, syntax, intonation, the subject of the discourse, the relationships between participants, or the communication channel. A fine language register study has not been on the scope of the work presented here, but will be considered for future work.

Currently, the utterance selection depending on the goals of the speaker is supposed to be held by an inference engine. We want to work on this inference engine, since it is possible to do meta-reasoning on emotions and social relations. For example, if a speaker wants to be liked by his interlocutor, knows that an utterance will cause joy emotion in the interlocutor, and knows this will lead the interlocutor to like him, he will use this utterance to achieve his goal of being liked. The inference engine should also determine if the emotion of fear induce a flight or fight behaviour. The current model only uses dominance to this purpose, but this is not a sufficient criteria. We plan to study emotional responses in a more detailed way, and beyond utterance selection, we hope to extend our model to general behaviour selection.

\section{ACKNOWLEDGMENTS}

We thank Vincent Corruble and Magalie Ochs for their contribution to our work, as Florent Boutin, Christophe Gohmann, and Karim Sehaba for their previous work on the DEEP project.

\section{REFERENCES}

[1] E. André, M. Rehm, W. Minker, and D. Bühler. Endowing spoken language dialogue systems with emotional intelligence. In Proceedings Affective Dialogue Systems 2004, pages 178-187. Springer, 2004.

[2] P. Brown and S. Levinson. Politeness, some universals in language usage. Cambridge University Press, 1987.

[3] J. Culpeper. Towards an anatomy of impoliteness. Journal of Pragmatics, 25:349-367, 1996.

[4] M. A. Walker, J. E. Cahn, and S. J. Whittaker. Improvising linguistic style: Social and affective bases for agent personality. In Proceedings of the First International Conference on Autonomous Agents (Agents'97), pages 96-105. ACM Press, 1997. 